\title{
Performance Evaluation of Exotic and Local Landraces of Tomatoes for the Mid-Hill Conditions of Nepal
}

\author{
Tek Prasad Gotame ${ }^{1, a, *}$, Ishwori Prasad Gautam ${ }^{1, b}$, Dipendra Ghimire ${ }^{1, c}$, Surendra Lal Shrestha ${ }^{1, d}$ \\ ${ }^{I}$ Nepal Agricultural Research Council, National Horticulture Research Centre, Khumaltar, Lalitpur, 1126 Kathmandu, Nepal \\ *Corresponding author
}

A R T I C L E IN F O A B S T R A C T

Research Article

The productivity of tomato in Nepal is very low due to lack of high yielding, disease and pests resistant varieties. An experiment was carried out to evaluate horticultural traits of 50 genotypes obtained from World Vegetable Centre (WorldVeg), Taiwan and SAARC region, and local collections during March to August 2020 in open field conditions at National Horticulture Research

Received : 09/02/2021

Accepted : 15/07/2021 Centre, Khumaltar, Lalitpur. The objectives were to identify promising open-pollinated tomato cultivars for high yield, appropriate fruit size, and disease resistant. The experiment was carried out in a randomized complete block design with three replications. Results showed significant differences in yield and yield attributing characters including virus infection. The highest yield (39.6 $\mathrm{mt} \mathrm{ha}^{-1}$ ) was produced by HRA43 and it was followed by HRA33 (26.4 $\left.\mathrm{mt} \mathrm{ha}^{-1}\right)$. Among the

Keywords:

Tomato

Fruit yield

WorldVeg OP lines

Local landraces

Cluster analysis WorldVeg OP lines, AVTO1429 produced the highest yield $\left(16.21 \mathrm{mt} \mathrm{ha}^{-1}\right)$ and it was followed by AVTO1717 (12.95 $\left.\mathrm{mt} \mathrm{ha}^{-1}\right)$, AVTO0922 (11.83 $\left.\mathrm{mt} \mathrm{ha}^{-1}\right)$ and AVTO1219 $\left(11.7 \mathrm{mt} \mathrm{ha}^{-1}\right)$ respectively. Most of the WorldVeg lines performed better than the check variety 'Pusa Ruby'. Genotype HRA43, Red Local and Sindhupalchock Local were not affected by virus while Yellow Local showed 3.3\% infection. Among the WorldVeg lines, AVTO1712 (20\%), AVTO1717 (20\%) and AVTO1718 (13\%) and AVTO1219 (15\%) showed less than 20\% virus infection in the open field conditions. Cluster analysis using the unweighted paired group method with arithmetic mean showed that cluster-1 was the largest cluster comprised of 40 genotypes followed by cluster- 2 and cluster-4. Genotypes from cluster-4 showed the higher fruit yield $\left(25.1 \mathrm{mt} \mathrm{ha}^{-1}\right)$ and resistant to the virus and the highest number of fruits per plot (1978 in $4.5 \mathrm{~m}^{2}$ area). The yield was low in cluster1 which could be due to the heavy rainfall during the vegetative and reproductive stages. Genotypes Red Local and Sindhupalchock Local could be used in future tomato breeding program due to their resistant to TYLCV, higher potential yield and highest plant vigour in open field conditions at the mid-hill of Nepal. Considering the overall performance, genotype HRA43, HRA33 and AVTO1429 were promising lines with performance for yield and other horticultural traits.

(iD https://orcid.org/0000-0003-0041-1363 (iD) https://orcid.org/0000-0002-8196-4017 Qshsurendra@hotmail.com https://orcid.org/0000-0003-0682-9306 https://orcid.org/0000-0003-1109-6514

\section{Introduction}

Tomato (Solanum lycopersicum $\mathrm{L} .2 \mathrm{n}=2 \mathrm{x}=24$ ) is one of the most widely grown vegetables in tropics and subtropics in the world. The total area under tomato cultivation is above 5 million ha with 180 million tonnes of production in the world (FAO, 2019). Tomato is the third largest vegetable crop in Nepal. It covers 22,566 ha with a total production of 406,434 mt (MoALD, 2019). Due to the lack of abiotic and biotic stress tolerant tomato varieties, the productivity is low in Nepal (18.01 $\mathrm{mt} \mathrm{ha}^{-1}$ ) (MoALD, 2019) as compared to India (24.33 $\left.\mathrm{mt} \mathrm{ha}^{-1}\right)$, Asia (43.10 mt $\mathrm{ha}^{-1}$ ) and the world (35.90 $\left.\mathrm{mt} \mathrm{ha}^{-1}\right)$ (FAO, 2019). The main reasons of low productivity of tomato in Nepal are due to the lack of suitable varieties resistance to diseases and pest, flower drop and poor fruiting, poor quality fruits particularly when the tomato is grown during the rainy season in open field conditions (Paudel et al., 2004). Unavailability of quality hybrid seeds may force the farmers for cultivation of open-pollinated cultivars (Shrestha and Sah, 2014). Imported hybrids are not available in the seed supply system over the years while Nepal cannot produce seeds of these hybrids due to lack of indreds for seed production.

In contrary, it has been observed that many promising local selections of tomato are popular but they are not coming in the national mainstreaming because of unavailability of seed in the national seed system. As 
hybrids are more vigorous, resistant to insect pests and diseases, high yielding, its use is increasing both commercial and small growers. The continuous inflow of exotic hybrid varieties and risk of getting appropriate variety at the right season and place is a great problem to the growers (Devkota et al., 2018). Hybrid seeds in vegetable crops are importing to Nepal from abroad especially from India, Thailand, Japan, and Korea. It is necessary to develop tomato OP varieties to replace the imported hybrid seeds and availability of sufficient quality seeds in the market.

Research in variety development in tomato has been initiated in Nepal since 1996 (Bhattarai and Subedi, 1996; Budhathoki et al., 2004; Paudel et al., 2004; Regmi et al., 2004). Budhathoki et al. (2004) evaluated 51 tomato genotypes under the open field of Khumaltar conditions, and recommended hybrids 'Swarachha', 'Manisha', 'T 5975' and 'NS 815' for cultivation. Paudel et al. (2004) evaluated 16 genotypes and found that N-162 was the highest yielding, and bacterial wilt resistant in the open field conditions of mid-hills. Devkota et al. (2018) evaluated hybrid cultivars at mid-hill conditions of Nepal (Khumaltar) and found that 'HRA14 x HRD7' produced the highest yield $\left(80.8 \mathrm{mt} \mathrm{ha}^{-1}\right)$. Chapagain et al. (2014) evaluated some exotic hybrids and recorded 'Winsari' the highest yielding (105.8 $\left.\mathrm{mt} \mathrm{ha}^{-1}\right)$ cultivar. However, these exotic varieties may not give stable performance over the season, seeds of these varieties are not available in the market and most of these varieties are not registered in Nepal. Their parental lines are unknown and need to depend to neighboring countries. Therefore, continuous OP varietal evaluation is necessary for providing sufficient varietal options for the farmers. Nepal Agricultural Research Council is keen to promote high yielding, disease resistant OP varieties so that famers can produce seeds. Both local and exotic tomato genotypes are a rich pool of genetic resources and can be used in tomato variety development program. Research is necessary to identify suitable variety at multi-location conditions such as an open field or protected cultivation system; mid-hills or Terai conditions. Therefore, this experiment was carried out to overcome these problems and to develop high yielding and disease-resistant open-pollinated varieties for the mid-hills of Nepal.

\section{Materials and Methods}

A total of 50 germplasms were collected both from national and international sources particularly from World Vegetable Center, Taiwan including SAARC region. Five local landraces were collected from farmer's field and included in the evaluation (Table 1).

The seeds of collected tomato germplasms were sown in poly-pot on February 26, 2020. The experiment was carried out in a randomized complete block design with three replications. Individual plant size was $4.5 \mathrm{~m}^{2}$ with two rows having 10 plants in each plot. Manure and fertilizers were applied at the rate of $25 \mathrm{mt} \mathrm{ha}^{-1} \mathrm{FYM}$ and 200:150:120 kg N: $\mathrm{P}_{2} \mathrm{O}_{5}: \mathrm{K}_{2} \mathrm{O}$ per hectare. i.e., $1.08 \mathrm{~kg}$ farmyard manure (FYM), $10.15 \mathrm{~g}$ urea (46\% nitrogen), $14.08 \mathrm{~g}$ di-ammonium phosphate (DAP; $18 \%$ nitrogen and $46 \%$ phosphorus) and $4.8 \mathrm{~g}$ muriate of potash (MoP) per plot. All FYM, $\mathrm{P}_{2} \mathrm{O}_{5}: \mathrm{K}_{2} \mathrm{O}$ and half of $\mathrm{N}$ were applied before one week of transplanting of seedlings. About 28 days old seedlings were transplanted in the open field in March 2020. Planting was done at a spacing of $75 \mathrm{~cm} \mathrm{x} 60 \mathrm{~cm}$ distance. The remaining half dose of nitrogen was equally top-dressed at 30 and 60 days after transplanting. The source of the fertilizer was urea which was applied at the rate of $5 \mathrm{~g}$ per plant. The plant extract, Vircon $\mathrm{H}$ was sprayed at the rate of $2 \mathrm{~mL} \mathrm{~L}^{-1}$ water as a protective spray against viruses. For control of late blight, systematic fungicide Dimethomorph 50\% WP (Acrobat) was sprayed at the rate of $1.5 \mathrm{~g} \mathrm{~L}^{-1}$ water. The protective fungicides Krinoxyl Gold Mz 72\% (metalaxyl 8\% + mancozeb 64\%) was sprayed 3 times at the rate of $2 \mathrm{~g} \mathrm{~L}^{-1}$ water at 10 days intervals to manage late blight and other fungal diseases. A micronutrient, Multiplex at the rate of $2 \mathrm{~mL} \mathrm{~L}^{-1}$ water was foliar sprayed along with the first spay of Krinoxyl Gold. Other intercultural operations as staking, earthing-up and weeding were carried out as per recommendation. Removal of suckers and removing of dead leaves was done first time on May 2020 and regularly at 7 days intervals.

\section{Observations}

Plant Vigour

Vigour was recorded at about 50\% flowering stage using a 1 to 5 rating scales methods described by Gotame et al. $(2019,2021)$.

- Very week (all plants were small, few leaves, week plants, very thin stems and light green colour)

- Week (75\% of the plants were small or all plants were shorter than normal plant height, plants have few leaves, thin stem and light green colour)

- Medium, intermediate or normal growth

- Vigorous (75\% of the plants were taller than normal, robust with foliage of dark green colour, thick stems and leaves were well developed)

- Very vigorous (all plants were taller than normal, ground completely covered by foliage, plants were robust, with a thick stem and abundant foliage of dark green colour)

\section{Days to $50 \%$ Flowering}

The number of days after transplanting (DAT) to $50 \%$ anthesis (50\% of plants in a plot have open flowers) was recorded. Each plot was checked three times a week and recorded the data.

\section{Growth Habit}

Growth habit was evaluated based on three types of growth habits as methods described by Hanson et al. (2013) and Gotame et al. (2019).

Determinate (D): Short and bushy, producing two leaves between flower clusters and about five clusters per branch were recorded as the determinant. They produced most of the fruits on terminal end and stopped to further growth and flowering. In this type, the number of leaves or internodes between inflorescences was varied from one to three.

Semi-determinate (SD): It lies in between determinate and indeterminate. They were capable of producing fruits longer period. Plants were more compact but less tall than indeterminate type. 
Indeterminate (ID): Tall, producing three leaves between flower clusters and more than six clusters per branch were recorded as indeterminate. The terminal axes did not stop to grow and flowering occurred from auxiliary buds.

Evaluation of Genotypes Against Tomato Yellow Leaf Curl Virus (TYLCV)

The individual plot was visited, monitored the appearance of the individual plant at least weekly early in the morning, to observe virus diseased plants, and counted the numbers of infected plants per plot in each observation under natural field conditions. Data were recorded on the data sheet as methods described by Hanson et al. (2013) and Gotame et al. (2019).

Tomato yellow leaf curl virus infection (TYLCV) \% was computed as virus incidence percent as:

$$
\text { Virus infection } \%=\frac{\text { Number of virüs-infected plants }}{\text { Total number of plants in a plot }} \times 100
$$

Table 1. Name and source of tomato germplasms/lines used in the experiment

\begin{tabular}{|c|c|c|c|c|}
\hline$\overline{\mathrm{SN}}$ & Germplasms & Growth habit & Year of introduction/collection & Source of introduction \\
\hline$\overline{1}$ & AVTO0102 & $\mathrm{D}^{1}$ & 2020 & World Vegetable Center, Taiwan \\
\hline 2 & AVTO0301 & $\mathrm{D}$ & 2020 & World Vegetable Center, Taiwan \\
\hline 3 & AVTO0922 & $\mathrm{D}$ & 2019 & World Vegetable Center, Taiwan \\
\hline 4 & AVTO1008 & $\mathrm{D}$ & 2019 & World Vegetable Center, Taiwan \\
\hline 5 & AVTO1288 & $\mathrm{D}$ & 2020 & World Vegetable Center, Taiwan \\
\hline 6 & AVTO1219 & $\mathrm{SD}^{2}$ & 2019 & World Vegetable Center, Taiwan \\
\hline 7 & AVTO1306 & SD & 2020 & World Vegetable Center, Taiwan \\
\hline 8 & AVTO1314 & SD & 2020 & World Vegetable Center, Taiwan \\
\hline 9 & AVTO1315 & SD & 2020 & World Vegetable Center, Taiwan \\
\hline 10 & AVTO1350 & $\mathrm{D}$ & 2019 & World Vegetable Center, Taiwan \\
\hline 11 & AVTO1409 & $\mathrm{D}$ & 2020 & World Vegetable Center, Taiwan \\
\hline 12 & AVTO1418 & $\mathrm{D}$ & 2019 & World Vegetable Center, Taiwan \\
\hline 13 & AVTO1420 & $\mathrm{D}$ & 2019 & World Vegetable Center, Taiwan \\
\hline 14 & AVTO1422 & $\mathrm{D}$ & 2019 & World Vegetable Center, Taiwan \\
\hline 15 & AVTO1424 & SD & 2020 & World Vegetable Center, Taiwan \\
\hline 16 & AVTO1429 & SD & 2019 & World Vegetable Center, Taiwan \\
\hline 17 & AVTO1464 & $\mathrm{ID}^{3}$ & 2020 & World Vegetable Center, Taiwan \\
\hline 18 & AVTO1616 & SD & 2020 & World Vegetable Center, Taiwan \\
\hline 19 & AVTO1702 & SD & 2020 & World Vegetable Center, Taiwan \\
\hline 20 & AVTO1705 & $\mathrm{D}$ & 2020 & World Vegetable Center, Taiwan \\
\hline 21 & AVTO1711 & $\mathrm{D}$ & 2020 & World Vegetable Center, Taiwan \\
\hline 22 & AVTO1712 & $\mathrm{D}$ & 2020 & World Vegetable Center, Taiwan \\
\hline 23 & AVTO1713 & $\mathrm{D}$ & 2020 & World Vegetable Center, Taiwan \\
\hline 24 & AVTO1715 & $\mathrm{D}$ & 2020 & World Vegetable Center, Taiwan \\
\hline 25 & AVTO1716 & $\mathrm{D}$ & 2020 & World Vegetable Center, Taiwan \\
\hline 26 & AVTO1717 & $\mathrm{D}$ & 2020 & World Vegetable Center, Taiwan \\
\hline 27 & AVTO1718 & SD & 2020 & World Vegetable Center, Taiwan \\
\hline 28 & AVTO1719 & SD & 2020 & World Vegetable Center, Taiwan \\
\hline 29 & AVTO1720 & SD & 2020 & World Vegetable Center, Taiwan \\
\hline 30 & AVTO9802 & $\mathrm{D}$ & 2019 & World Vegetable Center, Taiwan \\
\hline 31 & CL-1131 & SD & 1981 & World Vegetable Center, Taiwan \\
\hline 32 & CLN2545B & $\mathrm{D}$ & 2007 & World Vegetable Center, Taiwan \\
\hline 33 & HRA33 & ID & 2008 & \\
\hline 34 & HRA34 & ID & 2008 & \\
\hline 35 & HRA43 & ID & 2008 & \\
\hline 36 & HRD109 & ID & Unknown & Nepal \\
\hline 37 & HRD1455 & ID & 2017 & Nepal \\
\hline 38 & HRD9331 & ID & 2017 & Nepal \\
\hline 39 & HRD9708 & $\mathrm{D}$ & 2017 & Nepal \\
\hline 40 & HRDTOM011 & ID & 2004 & India \\
\hline 41 & HRDTOM079 & ID & 2013 & SAARC \\
\hline 42 & HRDTOM080 & ID & 2013 & SAARC \\
\hline 43 & HRDTOM083 & $\mathrm{D}$ & 2013 & SAARC \\
\hline 44 & HRDTOM085 & SD & 2013 & SAARC \\
\hline 45 & Pusa Ruby & $\mathrm{D}$ & 1981 & World Vegetable Center, Taiwan \\
\hline 46 & Red Local & ID & 2020 & Sindhupalchock, Nepal \\
\hline 47 & Sarlahi Lapsigede & $\mathrm{D}$ & 2020 & Sarlahi, Nepal \\
\hline 48 & Sarlahi Purbely Chiuri & $\mathrm{D}$ & 2020 & Sarlahi, Nepal \\
\hline 49 & Sindhupalchock Local & ID & 2020 & Sindhupalchock, Nepal \\
\hline 50 & Yellow Local & ID & 2020 & Sindhupalchock, Nepal \\
\hline
\end{tabular}


Table 2. Maximum and minimum temperature and rainfall during the tomato growing period

\begin{tabular}{l|ccc}
\hline \multicolumn{1}{c|}{ Month } & Maximum temp $\left({ }^{\circ} \mathrm{C}\right)$ & Minimum temp $\left({ }^{\circ} \mathrm{C}\right)$ & Rainfall $(\mathrm{mm})$ \\
\hline 15-March & 21.23 & 7.56 & 24.1 \\
30-March & 23.98 & 9.77 & 9.2 \\
15-April & 26.52 & 10.28 & 5.0 \\
30-April & 24.73 & 13.33 & 89.8 \\
15-May & 24.93 & 14.70 & 62.9 \\
30-May & 28.07 & 17.77 & 87.8 \\
15-June & 27.31 & 18.77 & 82.6 \\
30-June & 26.97 & 20.83 & 186.0 \\
15-July & 28.07 & 20.93 & 115.0 \\
30-July & 26.79 & 20.57 & 273.0 \\
15-August & 29.13 & 21.48 & 132.3 \\
30-August & 28.48 & 21.04 & 48.9 \\
\hline
\end{tabular}

\section{Yield and Yield Attributing Parameters}

Total number of fruits per plot, total yield per plot $(\mathrm{kg})$ and fruit size $(\mathrm{g})$ were recorded at each harvesting time. There were 7 harvestings of the fruits.

\section{Number of Fruits Per Plant}

Number of fruits per plant was recorded in each harvest lot separately and cumulative number of fruits was obtained by adding after the complete harvest.

\section{Fruit Weight $(g)$}

Average fruit weight $(\mathrm{g})$ was measured from randomly selected 20 marketable fruits per plot. The weight was recorded in two times, first at third and second at fifth harvest lot.

\section{Fruit Length and Diameter ( $\mathrm{mm}$ )}

Fruit length and diameter were measured by using a vernier caliper in randomly selected 20 marketable fruits harvested at third and fifth lot.

Fruit shape was recorded as described in IPGRI (1996) and Gotame et al. (2019).

- $\quad$ Flattened (oblate)

- $\quad$ Slightly flattened

- $\quad$ Rounded

- High rounded

- Heart-shaped

- Cylindrical (long oblong)

- $\quad$ Pyriform

- Ellipsoid

- Other

Total Soluble Solid $\left({ }^{\circ} \mathrm{B}\right)$

The total soluble solids of the selected samples were determined with a hand refractometer, Model ATAGO, Tokyo, Japan $\left(0-32^{\circ}\right.$ Brix range). The refractometer was washed with distilled water each time after use and dried with blotting paper to avoid contamination.

Weather Data

The weather data during the crop period (March to August 2020) were obtained from the National Agronomy Research Centre, Khumaltar, Lalitpur. The rainfall ranges from 24.1 to $132.3 \mathrm{~mm}$ from 15 March to 15 August. But there was heavy rainfall during the flowering and fruiting period (i.e., June-July, 2020) ranging from $82.6 \mathrm{~mm}$ in June to $273 \mathrm{~mm}$ in July. The continuous heavy rainfall during mid-April $(89.8 \mathrm{~mm})$ to end of May $(82.6 \mathrm{~mm})$ was very unusual in Nepal (Table 2).

\section{Data Analysis}

The experimental data were processed by using MS Excel 2016 and analysed by using Genstat var. 18.0 (GenStat). Two-way ANOVA for F-test for RCBD design was used to analyse the differences between the means observed parameters on growth, yield and yield attributing characters, and virus. Normality was checked using histogram before analysis. Clustering observations unweighted paired group method with arithmetic mean (UPGMA) clustering divided 50 lines of tomato into five clusters by using Minitab version 17. Pearson's correlation analysis between 9 quantitative traits was carried out by using Minitab version 17.

\section{Results and Discussion}

\section{Morphological Traits}

Highly significant differences were observed in days to $50 \%$ flowering, plant vigour, yield and yield attributing parameters and virus incidence (Table $3 a, 3 b$ and Table 4). Most of the lines received from WorldVeg performed better than the check variety Pusa Ruby. Pusa Ruby is an established and released variety of tomato in Nepal.

Plant vigour is a significant factor of tomato that affects yield attributing parameters and helps towards the final yield. In our study, plant vigour was highly significantly different between genotypes which could be due to genotypic variations existed in the tomatoes (Gurung et al., 2020; Gotame et al., 2021). Genotypes Red Local and Sindhupalchok Local were found to be highly vigorous (4.5 out of 1-5 scale). Genotypes HRA43, HRD109 and HRD1455 were also found to be vigorous with a vigour score of 4.33 out of $1-5$ scale. Days to $50 \%$ flowering was also highly significantly different between genotypes. Sarlahi Purbely Chiuri, Sindhupalchok Local and HRA34 were the earliest genotype which showed 50\% flowering at 26 days after transplanting while AVTO1420 was the late (44 days after transplanting) genotype.

Genotypes HRA43, Red Local and Sindhupalchock Local were not affected by tomato yellow leaf curl virus (TYLCV) while Yellow Local showed 3.3\% infection with the virus. Among the WorldVeg lines, AVTO1718, AVTO1219, AVTO0922, and national collections HRD1455, HRDTOM035 and Yellow Local showed less than $14 \%$ TYLCV infection in the open field conditions. These genotypes could be promising genotypes for the future tomato breeding program in the country. 
In tomato, the most easily visible traits are fruit size and shape. The highest fruit length and diameter was found in AVTO1409 (50.02 and $52.78 \mathrm{~mm}$ respectively). Sindhupalchock Local has the lowest fruit length $(12.9 \mathrm{~mm})$. The smallest diameter was found in Red Local $(17.39 \mathrm{~mm})$. Fruit shape is considered by the ratio of length and diameter and describes the overall shape. Based on the descriptor developed by IPGRI (1996), fruit shapes were varied from high-rounded, rounded, rounded slightly, flattened (oblate), slightly flattened, heart-shaped, ellipsoid (plum shaped) in our genotypes. This variation is genotypic variations. In our study, it varied from heart-shaped, rounded, flattened and ellipsoid. Many factors are controlling these traits probably depending on the genotypic background (Panthee and Gotame, 2020).

Table 3a. Morphological traits of exotic and local tomato germplasms at the mid-hill conditions of Nepal

\begin{tabular}{|c|c|c|c|}
\hline Genotypes & Days to $50 \%$ flowering & Plant vigour (1-5) scale & $\operatorname{TYLCV}^{\ddagger}(\%)$ \\
\hline$\overline{\mathrm{AVTO} 1288}$ & $33^{b-h}$ & $2.5^{\mathrm{a}-\mathrm{d}}$ & $56.67^{f-j}$ \\
\hline AVTO1314 & $36^{\mathrm{f}-\mathrm{k}}$ & $3.33^{\mathrm{b}-\mathrm{g}}$ & $36.67^{b-j}$ \\
\hline AVTO1315 & $40^{\mathrm{j}-\mathrm{o}}$ & $3.67^{\mathrm{c}-\mathrm{g}}$ & $58.12^{\mathrm{g}-\mathrm{j}}$ \\
\hline AVTO1464 & $35^{e-j}$ & $3.5^{\mathrm{b}-\mathrm{g}}$ & $45^{\mathrm{c}-\mathrm{j}}$ \\
\hline AVTO1702 & $32^{a-g}$ & $4^{\mathrm{e}-\mathrm{g}}$ & $26.6^{\mathrm{a}-\mathrm{h}}$ \\
\hline AVTO1705 & $34^{\mathrm{d}-\mathrm{i}}$ & $3.16^{\mathrm{a}-\mathrm{g}}$ & $66.67^{\mathrm{jk}}$ \\
\hline AVTO1409 & $36^{\mathrm{f}-\mathrm{k}}$ & $3.5^{\mathrm{b}-\mathrm{g}}$ & $46.67^{\mathrm{d}-\mathrm{j}}$ \\
\hline AVTO1306 & $38^{\mathrm{h}-\mathrm{n}}$ & $3.17^{\mathrm{a}-\mathrm{g}}$ & $33.33^{\mathrm{a}-\mathrm{j}}$ \\
\hline AVTO1711 & $27^{\mathrm{ab}}$ & $3.5^{\mathrm{b}-\mathrm{g}}$ & $23.33^{\mathrm{a}-\mathrm{g}}$ \\
\hline AVTO1712 & $28^{\mathrm{a}-\mathrm{d}}$ & $3.67^{\mathrm{c}-\mathrm{g}}$ & $20^{\mathrm{a}-\mathrm{e}}$ \\
\hline AVTO1715 & $30^{\mathrm{a}-\mathrm{f}}$ & $3.33^{\mathrm{b}-\mathrm{g}}$ & $40^{c-j}$ \\
\hline AVTO1716 & $35^{e-j}$ & $3.67^{\mathrm{c}-\mathrm{g}}$ & $28.15^{\mathrm{a}-\mathrm{i}}$ \\
\hline AVTO1717 & $30^{\mathrm{a}-\mathrm{f}}$ & $3.67^{\mathrm{c}-\mathrm{g}}$ & $20^{\mathrm{a}-\mathrm{e}}$ \\
\hline AVTO1718 & $30^{\mathrm{a}-\mathrm{f}}$ & $3.17^{\mathrm{a}-\mathrm{g}}$ & $13.33^{\mathrm{a}-\mathrm{d}}$ \\
\hline AVTO1719 & $33^{b-h}$ & $3^{a-f}$ & $43.33^{\mathrm{c}-\mathrm{j}}$ \\
\hline AVTO1720 & $32^{\mathrm{a}-\mathrm{g}}$ & $3.33^{\mathrm{b}-\mathrm{g}}$ & $40^{c-j}$ \\
\hline HRA33 & $31^{\mathrm{a}-\mathrm{g}}$ & $4.17^{e-g}$ & $23.33^{\mathrm{a}-\mathrm{g}}$ \\
\hline AVTO0301 & $40^{\mathrm{j}-\mathrm{o}}$ & $3.5^{\mathrm{b}-\mathrm{g}}$ & $21.43^{\mathrm{a}-\mathrm{f}}$ \\
\hline AVTO1616 & $41^{\mathrm{k}-\mathrm{o}}$ & $2.25^{\mathrm{a}-\mathrm{c}}$ & $91.66^{\mathrm{k}}$ \\
\hline AVTO1424 & $37^{\mathrm{g}-\mathrm{m}}$ & $2.33^{\mathrm{a}-\mathrm{c}}$ & $36.67^{b-j}$ \\
\hline AVTO1713 & $35^{e-j}$ & $3.17^{\mathrm{a}-\mathrm{g}}$ & $23.59^{\mathrm{a}-\mathrm{g}}$ \\
\hline AVTO0102 & $43^{\mathrm{m}-\mathrm{o}}$ & $1.83^{\mathrm{a}}$ & $62.59^{\mathrm{i}-\mathrm{k}}$ \\
\hline AVTO1422 & $45^{\circ}$ & $2.75^{\mathrm{a}-\mathrm{e}}$ & $41.66^{\mathrm{c}-\mathrm{j}}$ \\
\hline AVTO1219 & $43^{1-o}$ & $3.33^{\mathrm{b}-\mathrm{g}}$ & $15^{\mathrm{a}-\mathrm{e}}$ \\
\hline AVTO1350 & $33^{b-h}$ & $3.5^{\mathrm{b}-\mathrm{g}}$ & $36.67^{\mathrm{b}-\mathrm{j}}$ \\
\hline AVTO1429 & $30^{\mathrm{a}-\mathrm{f}}$ & $3.17^{\mathrm{a}-\mathrm{g}}$ & $36.67^{b-j}$ \\
\hline AVTO1420 & $44^{\text {no }}$ & $3.17^{\mathrm{a}-\mathrm{g}}$ & $61.85^{\mathrm{h}-\mathrm{k}}$ \\
\hline AVTO1418 & $32^{a-g}$ & $3.67^{\mathrm{c}-\mathrm{g}}$ & $60^{\mathrm{h}-\mathrm{k}}$ \\
\hline AVTO9802 & $36^{\mathrm{f}-\mathrm{k}}$ & $3.33^{\mathrm{b}-\mathrm{g}}$ & $50^{e-j}$ \\
\hline AVTO0922 & $28^{a-d}$ & $3.83^{\mathrm{d}-\mathrm{g}}$ & $13.33^{\mathrm{a}-\mathrm{d}}$ \\
\hline AVTO1008 & $40^{\mathrm{j}-\mathrm{o}}$ & $3^{\mathrm{a}-\mathrm{f}}$ & $43.33^{\mathrm{c}-\mathrm{j}}$ \\
\hline HRA43 & $37^{\mathrm{g}-1}$ & $4.33^{\mathrm{fg}}$ & $0^{\mathrm{a}}$ \\
\hline HRD1455 & $27^{\mathrm{ab}}$ & $4.33^{\mathrm{fg}}$ & $13.33^{\mathrm{a}-\mathrm{d}}$ \\
\hline HRD9331 & $30^{\mathrm{a}-\mathrm{f}}$ & $4^{\mathrm{e}-\mathrm{g}}$ & $16.67^{\mathrm{a}-\mathrm{e}}$ \\
\hline HRD9708 & $35^{e-j}$ & $3.5^{\mathrm{b}-\mathrm{g}}$ & $66.67^{\mathrm{jk}}$ \\
\hline Pusa Ruby (Check) & $36^{\mathrm{f}-\mathrm{k}}$ & $3.33^{\mathrm{b}-\mathrm{g}}$ & $43.33^{\mathrm{c}-\mathrm{j}}$ \\
\hline CL-1131 & $31^{\mathrm{a}-\mathrm{f}}$ & $4.17^{\mathrm{e}-\mathrm{g}}$ & $20^{\mathrm{a}-\mathrm{e}}$ \\
\hline HRDTOM011 & $39^{\mathrm{i}-\mathrm{o}}$ & $3.17^{\mathrm{a}-\mathrm{g}}$ & $20^{\mathrm{a}-\mathrm{e}}$ \\
\hline HRD109 & $35^{e-j}$ & $4.33^{\mathrm{fg}}$ & $20^{\mathrm{a}-\mathrm{e}}$ \\
\hline CL-2545B & $34^{\mathrm{d}-\mathrm{i}}$ & $3^{\mathrm{a}-\mathrm{f}}$ & $30^{\mathrm{a}-\mathrm{i}}$ \\
\hline HRDTOM080 & $43^{\mathrm{m}-\mathrm{o}}$ & $4^{e-g}$ & $20^{\mathrm{a}-\mathrm{e}}$ \\
\hline HRDTOM079 & $39^{\mathrm{i}-\mathrm{o}}$ & $3^{\mathrm{a}-\mathrm{f}}$ & $36.67^{\mathrm{b}-\mathrm{j}}$ \\
\hline HRDTOM083 & $29^{\mathrm{a}-\mathrm{e}}$ & $3.5^{\mathrm{b}-\mathrm{g}}$ & $50^{e-j}$ \\
\hline HRDTOM085 & $30^{\mathrm{a}-\mathrm{f}}$ & $4^{e-g}$ & $10^{\mathrm{a}-\mathrm{c}}$ \\
\hline HRA34 & $26^{\mathrm{a}}$ & $4.17^{\mathrm{e}-\mathrm{g}}$ & $16.67^{\mathrm{a}-\mathrm{e}}$ \\
\hline Yellow Local & $36^{\mathrm{f}-\mathrm{k}}$ & $4.167^{\mathrm{e}-\mathrm{g}}$ & $3.33^{\mathrm{ab}}$ \\
\hline Red Local & $30^{\mathrm{a}-\mathrm{f}}$ & $4.5^{\mathrm{g}}$ & $0^{\mathrm{a}}$ \\
\hline Sidhupalchock Local & $26^{\mathrm{a}}$ & $4.5^{\mathrm{g}}$ & $0^{\mathrm{a}}$ \\
\hline Sarlahi Lapsigede & $27^{\mathrm{a}-\mathrm{c}}$ & $2.83^{\mathrm{a}-\mathrm{e}}$ & $60^{h-k}$ \\
\hline Sarlahi Purbely Chiuri & $26^{\mathrm{a}}$ & $2.17^{\mathrm{ab}}$ & $60^{\mathrm{h}-\mathrm{k}}$ \\
\hline F-test & $* * *$ & $* * *$ & $* * *$ \\
\hline LSD & 4.88 & 1.13 & 28.60 \\
\hline $\mathrm{CV}(\%)$ & 8.8 & 20.3 & 51.8 \\
\hline
\end{tabular}


Table 3b. Morphological traits of exotic and local tomato germplasms at the mid-hill conditions of Nepal

\begin{tabular}{|c|c|c|c|}
\hline Genotypes & Fruit length $(\mathrm{mm})$ & Fruit diameter (mm) & Fruit shape \\
\hline AVTO1288 & $47.85^{\mathrm{i}-\mathrm{k}}$ & $43.51^{\mathrm{h}-\mathrm{o}}$ & $\mathrm{HR}^{1}$ \\
\hline AVTO1314 & $40.1^{\mathrm{c}-\mathrm{j}}$ & $41.76^{\mathrm{h}-\mathrm{n}}$ & Flattened \\
\hline AVTO1315 & $37.25^{\mathrm{c}-\mathrm{g}}$ & $38.39^{e-j}$ & Rounded \\
\hline AVTO1464 & $48.73^{\mathrm{jk}}$ & $50.05^{1-o}$ & Flattened \\
\hline AVTO1702 & $43.29^{\mathrm{d}-\mathrm{k}}$ & $45.8^{\mathrm{i}-\mathrm{o}}$ & Rounded \\
\hline AVTO1705 & $40.37^{\mathrm{c}-\mathrm{j}}$ & $39.48^{\mathrm{f}-\mathrm{k}}$ & $\mathrm{SF}^{2}$ \\
\hline AVTO1409 & $50.02^{\mathrm{k}}$ & $52.78^{\circ}$ & Rounded \\
\hline AVTO1306 & $49.86^{\mathrm{k}}$ & $46.38^{\mathrm{i}-\mathrm{o}}$ & Rounded \\
\hline AVTO1711 & $41.65^{\mathrm{c}-\mathrm{k}}$ & $39.95^{\mathrm{g}-\mathrm{k}}$ & Flattened \\
\hline AVTO1712 & $40.23^{\mathrm{c}-\mathrm{j}}$ & $51.35^{\text {no }}$ & Flattened \\
\hline AVTO1715 & $44.2^{\mathrm{e}-\mathrm{k}}$ & $47.95^{\mathrm{j}-\mathrm{o}}$ & Flattened \\
\hline AVTO1716 & $39.09^{c-i}$ & $47.15^{\mathrm{i}-\mathrm{o}}$ & Flattened \\
\hline AVTO1717 & $43.95^{\mathrm{e}-\mathrm{k}}$ & $48.51^{\mathrm{k}-\mathrm{o}}$ & Flattened \\
\hline AVTO1718 & $44.85^{\mathrm{f}-\mathrm{k}}$ & $48.9^{\mathrm{k}-\mathrm{o}}$ & Flattened \\
\hline AVTO1719 & $41.63^{\mathrm{c}-\mathrm{k}}$ & $46.94^{\mathrm{i}-\mathrm{o}}$ & Flattened \\
\hline AVTO1720 & $36.06^{\mathrm{c}-\mathrm{f}}$ & $43.69^{h-n}$ & Flattened \\
\hline HRA33 & $25.06^{\mathrm{b}}$ & $27.95^{\mathrm{bc}}$ & Rounded \\
\hline AVTO0301 & $46.92^{\mathrm{h}-\mathrm{k}}$ & $41.3 \mathrm{~h}^{\mathrm{i}-\mathrm{m}}$ & HR \\
\hline AVTO1616 & $37.22^{\mathrm{c}-\mathrm{g}}$ & $40.66^{\mathrm{g}-1}$ & Rounded \\
\hline AVTO1424 & $45.98^{\mathrm{g}-\mathrm{k}}$ & $44.66^{\mathrm{i}-\mathrm{o}}$ & HR \\
\hline AVTO1713 & $45.85^{\mathrm{g}-\mathrm{k}}$ & $48.14^{\mathrm{j}-\mathrm{o}}$ & Flattened \\
\hline AVTO0102 & $42.09^{c-k}$ & $41.05^{\mathrm{h}-1}$ & Rounded \\
\hline AVTO1422 & $45.66^{\mathrm{g}-\mathrm{k}}$ & $44.52^{\mathrm{i}-\mathrm{o}}$ & HR \\
\hline AVTO1219 & $45.72^{\mathrm{g}-\mathrm{k}}$ & $46^{\mathrm{i}-\mathrm{o}}$ & HR \\
\hline AVTO1350 & $39.29^{c-i}$ & $42.96^{\mathrm{h}-\mathrm{o}}$ & SF \\
\hline AVTO1429 & $48.06^{\mathrm{i}-\mathrm{k}}$ & $42.02^{\mathrm{h}-\mathrm{n}}$ & $\mathrm{HS}^{3}$ \\
\hline AVTO1420 & $41.76^{\mathrm{c}-\mathrm{k}}$ & $41.99^{\mathrm{h}-\mathrm{n}}$ & Rounded \\
\hline AVTO1418 & $44.22^{\mathrm{e}-\mathrm{k}}$ & $44.49^{\mathrm{i}-\mathrm{o}}$ & Rounded \\
\hline AVTO9802 & $43.61^{\mathrm{e}-\mathrm{k}}$ & $41.43^{\mathrm{h}-\mathrm{n}}$ & Rounded \\
\hline AVTO0922 & $49.43^{\mathrm{k}}$ & $51.26^{\mathrm{m}-\mathrm{o}}$ & Rounded \\
\hline AVTO1008 & $49.79^{k}$ & $43.64^{\mathrm{h}-\mathrm{o}}$ & HR \\
\hline HRA43 & $26.15^{\mathrm{b}}$ & $31.45^{\mathrm{c}-\mathrm{g}}$ & Rounded \\
\hline HRD1455 & $35.43^{\mathrm{c}-\mathrm{e}}$ & $41.36^{\mathrm{h}-\mathrm{m}}$ & Flattened \\
\hline HRD9331 & $35.96^{\mathrm{c}-\mathrm{f}}$ & $41.95^{\mathrm{h}-\mathrm{n}}$ & Flattened \\
\hline HRD9708 & $45.42^{\mathrm{g}-\mathrm{k}}$ & $41.61^{\mathrm{h}-\mathrm{n}}$ & HR \\
\hline Pusa Ruby (Check) & $36.07^{\mathrm{c}-\mathrm{f}}$ & $37.16^{\mathrm{c}-\mathrm{i}}$ & Flattened \\
\hline CL-1131 & $34.06^{c}$ & $37.6^{\mathrm{d}-\mathrm{i}}$ & Rounded \\
\hline HRDTOM011 & $42.58^{\mathrm{c}-\mathrm{k}}$ & $46.36^{\mathrm{i}-\mathrm{o}}$ & SF \\
\hline HRD109 & $41.49^{\mathrm{c}-\mathrm{k}}$ & $34.29^{\mathrm{c}-\mathrm{h}}$ & Ellipsoid \\
\hline CL-2545B & $48.6^{\mathrm{jk}}$ & $42.25^{\mathrm{h}-\mathrm{n}}$ & HR \\
\hline HRDTOM080 & $44.17^{\mathrm{e}-\mathrm{k}}$ & $44.66^{\mathrm{i}-\mathrm{o}}$ & Rounded \\
\hline HRDTOM079 & $45.41^{\mathrm{g}-\mathrm{k}}$ & $46.84^{\mathrm{i}-\mathrm{o}}$ & Rounded \\
\hline HRDTOM083 & $39.78^{c-j}$ & $37.75^{\mathrm{d}-\mathrm{i}}$ & Rounded \\
\hline HRDTOM085 & $34.38^{\mathrm{cd}}$ & $33.91^{\mathrm{c}-\mathrm{h}}$ & Rounded \\
\hline HRA34 & $26.51^{\mathrm{b}}$ & $29.45^{\mathrm{b}-\mathrm{e}}$ & Rounded \\
\hline Yellow Local & $15.05^{\mathrm{a}}$ & $21.48^{\mathrm{ab}}$ & Rounded \\
\hline Red Local & $17.24^{\mathrm{a}}$ & $17.39^{\mathrm{a}}$ & Rounded \\
\hline Sidhupalchock Local & $12.9^{\mathrm{a}}$ & $18.48^{\mathrm{a}}$ & Rounded \\
\hline Sarlahi Lapsigede & $37.98^{\mathrm{c}-\mathrm{h}}$ & $28.84^{\mathrm{b}-\mathrm{d}}$ & HR \\
\hline Sarlahi Purbely Chiuri & $34.4^{\mathrm{cd}}$ & $30.47^{\mathrm{c}-\mathrm{f}}$ & Rounded \\
\hline F-test & $* * *$ & $* * *$ & \\
\hline LSD & 7.20 & 7.94 & \\
\hline $\mathrm{CV}(\%)$ & 11.1 & 12 & \\
\hline
\end{tabular}

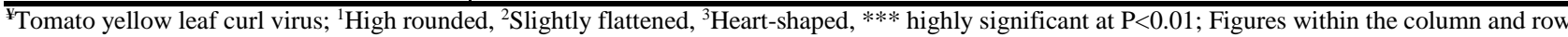
followed by same letters are not significantly different at $\mathrm{P}<0.05$ by DMRT

\section{Yield and Yield Attributing Traits}

Fruit weight plays an important role in yield. Within conditions, there was a significant difference in fruit weight (Table 4). In the present study, maximum fruit weight $(76.9 \mathrm{~g})$ was produced by AVTO1464 and it was followed by AVTO1409 (71.2 g) and AVTO1713 (70.6 g) while minimum fruit weight was recorded in Red Local
(3.7 g), Sindhupalchock Local (4.0 g) and Yellow Local $(6.2 \mathrm{~g})$. The variations in fruit weight may be attributed to inheritability of the genotypes.

Taste of tomato is determined by the ratio of total soluble solid (TSS) ( ${ }^{\circ}$ Brix) and titratable acidity. In our study, TSS varied from $2.26^{\circ}$ Brix (AVTO1720) to $5.23{ }^{\circ}$ Brix (AVTO1422). 
Table 4. Yield and yield attributing characters of exotic and local tomato germplasms at the mid-hill conditions of Nepal

\begin{tabular}{|c|c|c|c|c|c|}
\hline Genotypes & Fruit weight (g) & $\mathrm{TSS}^{\S}\left({ }^{\circ} \mathrm{Brix}\right)$ & Fruit no/plot $\left(4.5 \mathrm{~m}^{2}\right)$ & Fruit yield $\left(\mathrm{g} / 4.5 \mathrm{~m}^{2}\right)$ & Yield mt ha-1 \\
\hline AVTO1288 & $54^{\mathrm{i}-\mathrm{r}}$ & $4.5^{\mathrm{e}-\mathrm{j}}$ & $86^{\mathrm{a}}$ & $1642^{\mathrm{ab}}$ & $3.65^{\mathrm{ab}}$ \\
\hline AVTO1314 & $45.95^{\mathrm{g}-\mathrm{p}}$ & $3.5^{\mathrm{a}-\mathrm{i}}$ & $108^{\mathrm{a}}$ & $2782^{\mathrm{a}-\mathrm{e}}$ & $6.18^{\mathrm{a}-\mathrm{e}}$ \\
\hline AVTO1315 & $54.24^{\mathrm{i}-\mathrm{r}}$ & $4.533^{e-j}$ & $133^{\mathrm{a}}$ & $2669^{a-e}$ & $5.93^{\mathrm{a}-\mathrm{e}}$ \\
\hline AVTO1464 & $76.94^{\mathrm{s}}$ & $4.533^{e-j}$ & $77^{\mathrm{a}}$ & $3120^{\mathrm{a}-\mathrm{e}}$ & $6.93^{\mathrm{a}-\mathrm{e}}$ \\
\hline AVTO1702 & $43.84^{\mathrm{g}-\mathrm{o}}$ & $4.133^{b-j}$ & $127^{\mathrm{a}}$ & $2762^{\mathrm{a}-\mathrm{e}}$ & $6.14^{\mathrm{a}-\mathrm{e}}$ \\
\hline AVTO1705 & $46.89^{\mathrm{g}-\mathrm{p}}$ & $2.967^{\mathrm{a}-\mathrm{d}}$ & $187^{\mathrm{a}}$ & $3735^{\mathrm{a}-\mathrm{f}}$ & $8.30^{\mathrm{a}-\mathrm{f}}$ \\
\hline AVTO1409 & $71.2^{\mathrm{rs}}$ & $3.533^{\mathrm{a}-\mathrm{i}}$ & $146^{\mathrm{a}}$ & $4306^{\mathrm{a}-\mathrm{f}}$ & $9.57^{\mathrm{a}-\mathrm{f}}$ \\
\hline AVTO1306 & $60.26^{1-s}$ & $4^{b-j}$ & $132^{\mathrm{a}}$ & $3642^{\mathrm{a}-\mathrm{e}}$ & $8.09^{\mathrm{a}-\mathrm{e}}$ \\
\hline AVTO1711 & $52.06^{\mathrm{h}-\mathrm{r}}$ & $4.733^{\mathrm{h}-\mathrm{j}}$ & $119^{\mathrm{a}}$ & $3647^{\mathrm{a}-\mathrm{e}}$ & $8.10^{\mathrm{a}-\mathrm{e}}$ \\
\hline AVTO1712 & $64.31^{\mathrm{o}-\mathrm{s}}$ & $3.233^{\mathrm{a}-\mathrm{g}}$ & $132^{\mathrm{a}}$ & $3880^{\mathrm{a}-\mathrm{f}}$ & $8.62^{\mathrm{a}-\mathrm{f}}$ \\
\hline AVTO1715 & $61.38^{\mathrm{m}-\mathrm{s}}$ & $3.567^{\mathrm{a}-\mathrm{i}}$ & $73^{\mathrm{a}}$ & $2222^{\mathrm{a}-\mathrm{c}}$ & $4.94^{\mathrm{a}-\mathrm{c}}$ \\
\hline AVTO1716 & $66.56^{\mathrm{p}-\mathrm{s}}$ & $3.367^{\mathrm{a}-\mathrm{i}}$ & $69^{\mathrm{a}}$ & $2004^{\mathrm{ab}}$ & $4.45^{\mathrm{ab}}$ \\
\hline AVTO1717 & $58.46^{\mathrm{k}-\mathrm{s}}$ & $2.7^{\mathrm{ab}}$ & $156^{\mathrm{a}}$ & $5832^{\mathrm{b}-\mathrm{g}}$ & $12.96^{\mathrm{b}-\mathrm{g}}$ \\
\hline AVTO1718 & $63.89^{n-s}$ & $3.3^{\mathrm{a}-\mathrm{i}}$ & $164^{\mathrm{a}}$ & $4255^{\mathrm{a}-\mathrm{f}}$ & $9.46^{\mathrm{a}-\mathrm{f}}$ \\
\hline AVTO1719 & $49.94^{\mathrm{g}-\mathrm{q}}$ & $2.767^{\mathrm{ab}}$ & $102^{\mathrm{a}}$ & $2454^{\mathrm{a}-\mathrm{d}}$ & $5.45^{\mathrm{a}-\mathrm{d}}$ \\
\hline AVTO1720 & $49.75^{\mathrm{g}-\mathrm{q}}$ & $2.267^{\mathrm{a}}$ & $61^{a}$ & $1827^{\mathrm{ab}}$ & $4.06^{\mathrm{ab}}$ \\
\hline HRA33 & $13.67^{\mathrm{abc}}$ & $3.8^{\mathrm{b}-\mathrm{j}}$ & $1308^{\mathrm{c}-\mathrm{e}}$ & $11898^{\mathrm{h}}$ & $26.44^{\mathrm{h}}$ \\
\hline AVTO0301 & $58.68^{\mathrm{k}-\mathrm{s}}$ & $3.9^{b-j}$ & $118^{\mathrm{a}}$ & $2145^{\mathrm{a}-\mathrm{c}}$ & $4.77^{\mathrm{a}-\mathrm{c}}$ \\
\hline AVTO1616 & $41.56^{\mathrm{f}-\mathrm{m}}$ & $3.4^{\mathrm{a}-\mathrm{i}}$ & $40^{\mathrm{a}}$ & $668^{a}$ & $1.48^{\mathrm{a}}$ \\
\hline AVTO1424 & $57.09^{\mathrm{j}-\mathrm{s}}$ & $3.233^{\mathrm{a}-\mathrm{g}}$ & $85^{\mathrm{a}}$ & $1806^{\mathrm{ab}}$ & $4.01^{\mathrm{ab}}$ \\
\hline AVTO1713 & $70.65^{\text {qrs }}$ & $3.8^{\mathrm{b}-\mathrm{j}}$ & $82^{\mathrm{a}}$ & $3031^{\mathrm{a}-\mathrm{e}}$ & $6.74^{\mathrm{a}-\mathrm{e}}$ \\
\hline AVTO0102 & $44.97^{\mathrm{g}-\mathrm{o}}$ & $4.6^{\mathrm{f}-\mathrm{j}}$ & $105^{\mathrm{a}}$ & $1470^{\mathrm{ab}}$ & $3.27^{\mathrm{ab}}$ \\
\hline AVTO1422 & $57.48^{\mathrm{j}-\mathrm{s}}$ & $5.233^{\mathrm{j}}$ & $92^{\mathrm{a}}$ & $2437^{\mathrm{a}-\mathrm{d}}$ & $5.41^{\mathrm{a}-\mathrm{d}}$ \\
\hline AVTO1219 & $60.78^{1-s}$ & $3.25^{\mathrm{a}-\mathrm{g}}$ & $128^{\mathrm{a}}$ & $5260^{\mathrm{a}-\mathrm{g}}$ & $11.69^{\mathrm{a}-\mathrm{g}}$ \\
\hline AVTO1350 & $42.87^{\mathrm{f}-\mathrm{n}}$ & $3.567^{\mathrm{a}-\mathrm{i}}$ & $579^{a-d}$ & $3896^{\mathrm{a}-\mathrm{f}}$ & $8.66^{\mathrm{a}-\mathrm{f}}$ \\
\hline AVTO1429 & $55.48^{\mathrm{i}-\mathrm{r}}$ & $2.8^{\mathrm{a}-\mathrm{c}}$ & $269^{a}$ & $7288^{\mathrm{e}-\mathrm{g}}$ & $16.19^{\mathrm{e}-\mathrm{g}}$ \\
\hline AVTO1420 & $46.57^{\mathrm{g}-\mathrm{p}}$ & $3.167^{\mathrm{a}-\mathrm{f}}$ & $89^{a}$ & $2033^{\mathrm{a}-\mathrm{c}}$ & $4.52^{\mathrm{a}-\mathrm{c}}$ \\
\hline AVTO1418 & $49.91^{\mathrm{g}-\mathrm{q}}$ & $3.133^{\mathrm{a}-\mathrm{f}}$ & $171^{\mathrm{a}}$ & $3357^{\mathrm{a}-\mathrm{e}}$ & $7.46^{\mathrm{a}-\mathrm{e}}$ \\
\hline AVTO9802 & $49.92^{\mathrm{g}-\mathrm{q}}$ & $4.667^{\mathrm{g}-\mathrm{j}}$ & $136^{\mathrm{a}}$ & $2744^{\mathrm{a}-\mathrm{e}}$ & $6.10^{\mathrm{a}-\mathrm{e}}$ \\
\hline AVTO0922 & $57.97^{\mathrm{j}-\mathrm{s}}$ & $3.067^{\mathrm{a}-\mathrm{e}}$ & $197^{\mathrm{a}}$ & $5324^{\mathrm{a}-\mathrm{g}}$ & $11.83^{\mathrm{a}-\mathrm{g}}$ \\
\hline AVTO1008 & $48.99^{\mathrm{g}-\mathrm{p}}$ & $4.767^{\mathrm{ij}}$ & $152^{\mathrm{a}}$ & $3343^{a-e}$ & $7.43^{\mathrm{a}-\mathrm{e}}$ \\
\hline HRA43 & $16.89^{\mathrm{a}-\mathrm{d}}$ & $4.3^{\mathrm{d}-\mathrm{j}}$ & $3299^{g}$ & $17837^{\mathrm{i}}$ & $39.64^{\mathrm{i}}$ \\
\hline HRD1455 & $46.22^{\mathrm{g}-\mathrm{p}}$ & $3.1^{\mathrm{a}-\mathrm{e}}$ & $822^{\mathrm{a}-\mathrm{d}}$ & $9451^{\mathrm{gh}}$ & $21.01^{\mathrm{gh}}$ \\
\hline HRD9331 & $39.96^{\mathrm{f}-1}$ & $3.433^{\mathrm{a}-\mathrm{i}}$ & $526^{a b c}$ & $7112^{\mathrm{d}-\mathrm{g}}$ & $15.81^{\mathrm{d}-\mathrm{g}}$ \\
\hline HRD9708 & $47.75^{\mathrm{gp}}$ & $4 b^{c-j}$ & $116^{\mathrm{a}}$ & $1951^{\mathrm{ab}}$ & $4.34^{\mathrm{ab}}$ \\
\hline Pusa Ruby (Check) & $38.62^{\mathrm{f}-\mathrm{k}}$ & $4.667^{\mathrm{g}-\mathrm{j}}$ & $121^{\mathrm{a}}$ & $1342^{\mathrm{ab}}$ & $2.98^{\mathrm{ab}}$ \\
\hline CL-1131 & $31.88^{\mathrm{c}-\mathrm{h}}$ & $3.533^{\mathrm{a}-\mathrm{i}}$ & $408^{\mathrm{ab}}$ & $3480^{\mathrm{a}-\mathrm{e}}$ & $7.73^{\mathrm{a}-\mathrm{e}}$ \\
\hline HRDTOM011 & $53.76^{\mathrm{i}-\mathrm{r}}$ & $3.7^{\mathrm{a}-\mathrm{i}}$ & $122^{\mathrm{a}}$ & $3078^{a-e}$ & $6.84^{\mathrm{a}-\mathrm{e}}$ \\
\hline HRD109 & $30.66^{\mathrm{c}-\mathrm{g}}$ & $3.9^{b-j}$ & $1188^{\mathrm{b}-\mathrm{e}}$ & $9586^{\mathrm{gh}}$ & $21.30^{\mathrm{gh}}$ \\
\hline CL-2545B & $50.93^{\mathrm{g}-\mathrm{r}}$ & $4.433^{\mathrm{d}-\mathrm{j}}$ & $137^{\mathrm{a}}$ & $3202^{\mathrm{a}-\mathrm{e}}$ & $7.126^{\mathrm{a}-\mathrm{e}}$ \\
\hline HRDTOM080 & $53.48^{\mathrm{i}-\mathrm{r}}$ & $3.6^{\mathrm{a}-\mathrm{i}}$ & $270.3 \mathrm{a}$ & $7014^{\mathrm{d}-\mathrm{g}}$ & $15.59^{\mathrm{d}-\mathrm{g}}$ \\
\hline HRDTOM079 & $50.31^{\mathrm{g}-\mathrm{q}}$ & $3.367^{\mathrm{a}-\mathrm{i}}$ & $255^{\mathrm{a}}$ & $6749^{c-g}$ & $15.00^{c-g}$ \\
\hline HRDTOM083 & $35.45^{\mathrm{d}-\mathrm{i}}$ & $4.267^{c-j}$ & $106^{\mathrm{a}}$ & $2167^{\mathrm{a}-\mathrm{c}}$ & $4.82^{\mathrm{a}-\mathrm{c}}$ \\
\hline HRDTOM085 & $37.24^{e-j}$ & $3.267^{\mathrm{a}-\mathrm{h}}$ & $296^{\mathrm{a}}$ & $3653^{\mathrm{a}-\mathrm{e}}$ & $8.12^{\mathrm{a}-\mathrm{e}}$ \\
\hline HRA34 & $15.58^{\mathrm{a}-\mathrm{c}}$ & $3.467^{\mathrm{a}-\mathrm{i}}$ & $1691^{\text {ef }}$ & $8251^{\mathrm{f}-\mathrm{h}}$ & $18.34^{\mathrm{f}-\mathrm{h}}$ \\
\hline Yellow Local & $6.22^{\mathrm{ab}}$ & $3.6^{\mathrm{a}-\mathrm{i}}$ & $915^{\mathrm{a}-\mathrm{e}}$ & $2470^{\mathrm{a}-\mathrm{d}}$ & $5.49^{\mathrm{a}-\mathrm{d}}$ \\
\hline Red Local & $3.71^{\mathrm{a}}$ & $3^{\mathrm{a}-\mathrm{d}}$ & $2230^{f}$ & $4757^{\mathrm{a}-\mathrm{f}}$ & $10.57^{\mathrm{a}-\mathrm{f}}$ \\
\hline Sidhupalchock Local & $4.04^{\mathrm{a}}$ & $3.6^{\mathrm{a}-\mathrm{i}}$ & $1425^{\mathrm{de}}$ & $4018^{\mathrm{a}-\mathrm{f}}$ & $8.93^{\mathrm{a}-\mathrm{f}}$ \\
\hline Sarlahi Lapsigede & $18.72^{\mathrm{a}-\mathrm{e}}$ & $4.033^{\mathrm{b}-\mathrm{j}}$ & $204^{\mathrm{a}}$ & $2130^{\mathrm{a}-\mathrm{c}}$ & $4.73^{\mathrm{a}-\mathrm{c}}$ \\
\hline $\begin{array}{l}\text { Sarlahi Purbely } \\
\text { Chiuri }\end{array}$ & $23.77^{b-f}$ & $4^{b-j}$ & $238^{\mathrm{a}}$ & $2233^{\mathrm{a}-\mathrm{c}}$ & $4.96^{\mathrm{a}-\mathrm{c}}$ \\
\hline F-test & $* * *$ & $* * *$ & $* * *$ & $* * *$ & $* * *$ \\
\hline LSD & 16.82 & 1.16 & 751.3 & 3750.6 & 8.33 \\
\hline $\mathrm{CV}(\%)$ & 22.8 & 19.4 & 117.1 & 55.7 & 55.7 \\
\hline
\end{tabular}

§Total soluble solid, *** highly significant at $\mathrm{P}<0.01$; ns, non-significant at $\mathrm{P}>0.05$; Figures within the column and row followed by same letters are not significantly different at $\mathrm{P}<0.05$ by DMRT.

The highest yield (39.63 $\left.\mathrm{mt} \mathrm{ha}^{-1}\right)$ was produced by the genotype HRA43 and it was followed by HRA33 (26.40 mt $\mathrm{ha}^{-1}$ ). Among the WorldVeg lines, AVTO1429 produced the highest yield (16.21 $\left.\mathrm{mt} \mathrm{ha}^{-1}\right)$, followed by AVTO1717 (12.95 $\left.\mathrm{mt} \mathrm{ha}^{-1}\right)$, AVTO0922 (11.83 $\left.\mathrm{mt} \mathrm{ha}^{-1}\right)$ and AVTO1219 $\left(11.68 \mathrm{mt} \mathrm{ha}^{-1}\right)$. The yield was found to be low in most of the
WorldVeg lines which could be due to the heavy rainfall during the vegetative growth and fruiting period (Table 2). The check variety 'Pusa Ruby' produced fruit yield $2.98 \mathrm{mt}$ ha $^{-1}$ while most of the AVTO lines produced yield ranged from 1.48 to $16.19 \mathrm{mt} \mathrm{ha}^{-1}$. Yield, a complex character, is governed by many factors such as genotype, environment 
and management. Yield in each genotype is a result of the cumulative effect of different yield attributing characters. The poor yield of tomato in open field conditions was due to adverse weather which was un-predicted heavy rainfall during the flowering and fruiting period (April- May) (Table 2 ). The adverse weather condition was mostly associated with disease development. Even in such adverse open field conditions, three lines received from WorldVeg (AVTO1429, AVTO1717 and AVTO1219) were found to be promising in the mid-hill condition of Nepal due to higher yield and medium disease resistant (Table 2).

\section{Cluster Analysis}

Clustering observations Un-weighted paired group method with arithmetic mean (UPGMA) clustering divided
50 lines of tomato into five clusters in more than $80 \%$ similarity level (Figure 1).

Cluster-1 was the largest cluster comprised of 40 genotypes followed by cluster- 2 and cluster 3 comprised of 3 genotypes. Cluster 4 and cluster 5 comprised of 2 lines (Table 5). Cluster 4 comprised of 2 genotypes showed the highest fruit yield $\left(25.10 \mathrm{mt} \mathrm{ha}^{-1}\right)$ and the highest number of fruits per plot (1978 per $4.5 \mathrm{~m}^{2}$ area) (Table 5). Genotypes from this cluster could further be evaluated and used for future tomato breeding program. According to dendrogram Cluster 3 consisted of 3 genotypes (HRA33, Sidhupalchock Local and HRA34) and cluster 4 consisted up 2 genotypes (HRD109 and Yellow Local) could be useful genotypes due to their resistant to the virus, higher potential yield and highest plant vigour to develop high yielding genotypes for mid-hill conditions of Nepal.

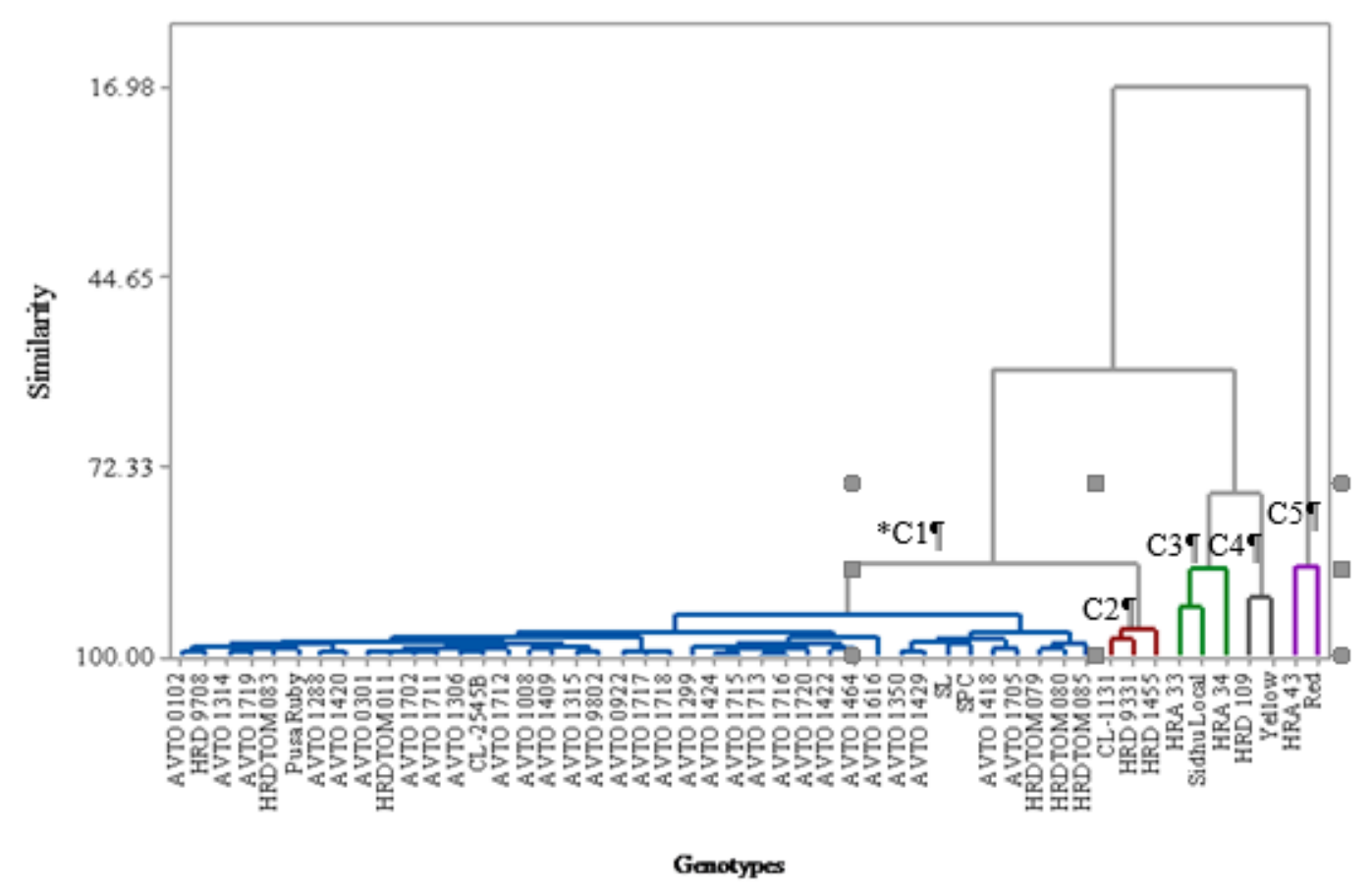

SL, Sarlahi Lapaigade; SPC, Sarlahi Purbeli Chaiuri; Red, Red Local; Yellow, Yellow Local; Sindlhndk Local, Sindhupaldhodk Local

Figure 1. Cluster analysis of tomato genotypes based on morphological traits *C1, Cluster 1; C2, Cluster 2; C3, Cluster 3; C4, Cluster 4; C5, Cluster 5

\section{Correlation Analysis}

The Pearson's correlation analysis between 10 quantitative traits showed that there was a positive correlation between growth habit and number of fruits per plot and yield per ha $(r=0.58$ and $r=0.53$ respectively). A highly significant positive correlation was observed between fruit weight $(\mathrm{g})$ with fruit length, fruit diameter $(\mathrm{r}=0.94)$ but there was a negative correlation with fruit weight and the number of fruits per plot $(\mathrm{r}=-0.76)$ (Table 6$)$.

Tomato genotypes behaved differently in morphological and physiological parameters (Rawal et al., 2017; Panthee and Gotame, 2020). The results of this study indicated significant differences among genotypic response towards growth and days to $50 \%$ flowering, virus infection, yield and yield attributing parameters which is in agreement with the findings of previous research reported by Shrestha and Sah (2014), and Gotame et al. (2021). Moreover, heavy rains and humid conditions result in poor flower development and low fruit set in tomato (Sato et al., 2000), which could be the reason for the low yield in our study. Wide variation has been observed in tomato yield among genotypes (Devkota et al., 2018). They found that yield variation from 54.39 to $80.83 \mathrm{mt} \mathrm{ha}^{-1}$ among 13 hybrids evaluated in the same field conditions at an altitude of $1350 \mathrm{~m}$ (mid-hills) of Nepal. Chapagain et al. (2014) reported tomato yield ranging from 71.4 to $105.8 \mathrm{mt} \mathrm{ha}^{-1}$ among six genotypes at an altitude of $1640 \mathrm{~m}$ (mid-hills) under plastic house conditions. In our open field study, the highest yield was produced by HRA43 (39.63 $\mathrm{mt} \mathrm{ha}^{-1}$ ) which was higher than the national average of the country (18.01 $\mathrm{mt} \mathrm{ha}^{-1}$ ) and comparable with world productivity (35.90 $\mathrm{mt} \mathrm{ha}^{-1}$ ). Generally, tomato yield increases two times under plastic house as compared to open field condition in mid-hills of Nepal. This suggests that yield is 
not only a genetic factor, it is also governed by the environment (Bhurtyal et al., 2007; Devkota et al., 2018).

Chapagain et al. (2020a) studied in tomatoes for fruit yield and yield contributing traits and were grouped into one cluster having taller plants with higher marketable fruits per plant, fruit set percentage, flowers per inflorescence, marketable fruits and fruit yield per plant. Tomato genotypes with semi-determinate to indeterminate growth habit with medium sized fruits performed better in yield and quality attributes (Chapagain et al., 2020b). It has been reported that the highest marketable fruit yield of 34.0 and $32.0 \mathrm{mt} \mathrm{ha}^{-1}$ was recorded in genotype AVTO9802 in 2018 and 2019, respectively with the mean yield of $32.9 \mathrm{mt}$ $\mathrm{ha}^{-1}$. In contrary to their report, the yield of AVTO lines in our study was low which was due to heavy rainfall and disease problem.

Chapagain et al. (2020a) found that the highest positive effect of marketable fruit per plant $(r=0.99)$ on fruit yield per plant. Correlation analysis revealed that plant height, fruit weight and diameter were positively associated with fruit yield (Reddy et al., 2013; Chapagain et al., 2020a). Chapagain et al. (2020a) reported that fruit diameter showed positive and significant correlation with average fruit weight (0.983). It was also found that there was strong positive correlation between fruit number per inflorescence and marketable fruit per plant. Bojarian et al. (2019) carried out path analysis and found that number of fruits per line and single fruit weight had the highest positive direct effect on yield.

A positive correlation of growth habit and plant vigour with the number of fruits per plot and fruit yield were observed in our study indicates the growth attributes are interlinked to maintain a higher and sustainable yield. Genotypes with indeterminate growth habit may have the ability to take up nutrients and water in amounts sufficient for the plant's growth and development, resulting in better vigour and yield as compared to determinant and semideterminate tomato. Similarly, fruit weight was highly and strongly correlated with fruit length and diameter. Balcha et al. (2015) found that fruit yield per hectare and fruit weight per plant was significantly and positively correlated. Fruit weight per plant was highly significantly and positively correlated with fruit yield per hectare. Reddy et al. (2013) also found that fruit yield per plant was positively and significantly correlated with number of fruits per plant and fruit width.

Table 5. Number of genotypes in each cluster and range of observations for morphological traits

\begin{tabular}{|c|c|c|c|c|c|}
\hline Traits & Cluster 1 & Cluster 2 & Cluster 3 & Cluster 4 & Cluster 5 \\
\hline Number of genotypes & 40 & 3 & 3 & 2 & 2 \\
\hline Growth habit & 1.5 & 2.7 & 3 & 3 & 3 \\
\hline Days to $50 \%$ flowering & 33 & 29 & 28 & 34 & 35 \\
\hline Plant vigour ( $1-5$ scale $)$ & 3.1 & 4.2 & 4.3 & 4.4 & 4.3 \\
\hline Fruit length (mm) & 43.1 & 35.1 & 21.4 & 21.7 & 28.3 \\
\hline Fruit diameter (mm) & 43.4 & 25.3 & 24.4 & 40.3 & 27.9 \\
\hline Fruit weight $(\mathrm{g})$ & 51.816 & 39.353 & 11.10 & 10.30 & 18.440 \\
\hline Total soluble solid ( ${ }^{\circ}$ Brix $)$ & 3.74 & 3.36 & 3.62 & 3.65 & 3.75 \\
\hline Number of fruits./plot $\left(4.5 \mathrm{~m}^{2}\right)$ & 134 & 411 & 1226 & 1978 & 727 \\
\hline $\operatorname{TLCMV}^{¥}(\%)$ & 38.1 & 16.7 & 13.33 & 0.0 & 11.7 \\
\hline Fruit yield (kg/plot) $\left(4.5 \mathrm{~m}^{2}\right)$ & 3.312 & 6.7 & 8.1 & 11.3 & 6.1 \\
\hline Yield $\left(\mathrm{mt} \mathrm{ha}^{-1}\right)$ & 7.8 & 14.8 & 17.9 & 25.1 & 13.4 \\
\hline
\end{tabular}

Growth habit, 1 = Determinate; $2=$ Semi-Determinate, and 3= Indeterminate, ${ }^{ }$Tomato leaf curl mosaic virus.

Table 6. Correlation analysis of morphological traits of tomato genotypes evaluated at the mid-hill condition of Nepal

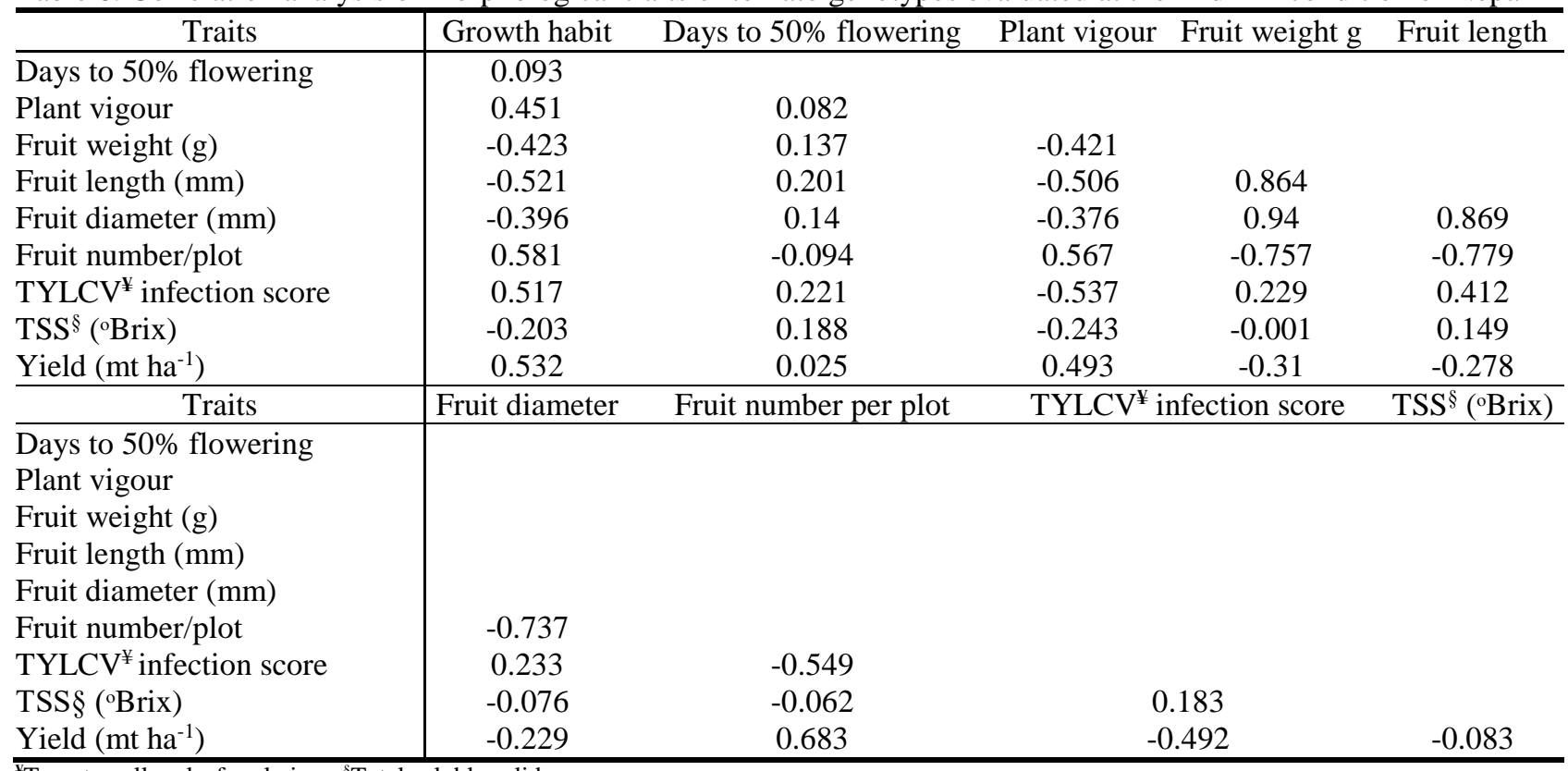

${ }^{\text {TT}}$ Tomato yellow leaf curl virus; ${ }^{\S}$ Total soluble solid 


\section{Conclusion}

Among the 50 evaluated genotypes, high yielding lines HRA43 (39.63 $\left.\mathrm{mt} \mathrm{ha}^{-1}\right)$ and HRA33 (26.43 $\mathrm{mt} \mathrm{ha}^{-1}$ ) could be the most potential varieties for an open field of the midhill of Nepal. As this study was carried out in open field in OP lines, yield is above average level as compared to other OP cultivars. Three lines received from WorldVeg were also found to be promising. These lines produced higher yield and showed disease resistant as compared to check variety Pusa Ruby in open field conditions. These lines were AVTO1429, AVTO1717 and AVTO1219. Genotypes HRA43, Red Local and Sindhupalchock Local could be used in future tomato breeding program due to their resistant to TYLCV. These landraces could be selected for further evaluation in the farmers' field and considered as important genetic resources to develop tomato varieties resistant to the virus at the mid-hill's region of the country. This study showed high agromorphological diversity among exotic and local landraces. We provide varietal options to farmers to produce tomato in mid-hill conditions of Nepal.

\section{Authors Contribution}

T.P. Gotame designed the experiment, wrote the draft and finalized the manuscript, I.P. Gautam designed, executed the experiment and finalized the initial draft, D. Ghimire involved in the field layout, genotype evaluation, data collection and analysis, and S.L. Shrestha executed the experiment and finalized the initial draft of the manuscript.

\section{Conflict of Interest}

The authors declare no conflicts of interest.

\section{Acknowledgement}

Acknowledgement goes to World Vegetable Centre, Taiwan for supplying the promising tomato germplasm/lines; AFACI, RDA, Korea for financial and technical support. Mr Krishna Hari Ghimire and Dr Mahendra Tripathi are highly acknowledged for helping the data analysis.

\section{References}

Balcha K, Belew D, Nego J. 2015. Evaluation of tomato (Lycopersicon esculentum Mill.) varieties for seed yield and yield components under Jimma Condition, South Western Ethiopia. Journal of Agronomy, 14(4): 292-297. doi: 10.3923/ja.2015.292.297

Bhattarai SP, Subedi PP. 1996. Assessment of tomato varieties for heat and bacterial wilt tolerant in low hills during 199293. LARC working paper No. 96/36. Lumle Agriculture Research Center, Kaski, Nepal.

Bhurtyal PR, Shakya SM, Dhakal DD, Upreti G. 2007. Evaluation of heat-tolerant tomato (Solanum lycopersicon) genotypes during summer in Chitwan, Nepal. IAAS Res. Advances, 2: 47-51.

Bojarian M, Asadi-Gharneh HA, Golabadi, M. 2019. Factor analysis, stepwise regression and path coefficient analyses of yield, yield-associated traits, and fruit quality in tomato. International Journal of Vegetable Science, 25: 542-553. doi:10.1080/19315260.2018.1551260
Budhathoki K, Pradan NG, Regmi HN, Bhurtayal PR. 2004. Evaluation of tomato cultivar for off-season production under the polyhouse and open field condition. Proceeding of the Fourth National Workshop on Horticulture, March 2-4, 2004. Nepal Agricultural Research Council, National Agriculture Research Institute and Horticulture Research Division, Khumaltar, Lalitpur, pp: 413-418.

Chapagain TR, Shrestha AK, Sharma MD, Tripathi KM, Srivastva A. 2020a. Evaluation and characterization of tomato (Solanum lycopersicum L.) genotypes using path coefficient, GGE biplot and cluster analyses under natural heat stress. International Journal of Horticulture, 10(6): 1-10. doi: 10.5376/ijh.2020.10.0006)

Chapagain T, Shrestha AK, Sharma MD, Mishra Tripathi K, Srivastva A. 2020b. Growth, yield and biochemical characteristics of tomato (Solanum lycopersicum L.) genotypes under seasonal heat stress. Asian J. Agric. Hortic. Res., 6(2): 22-29. doi: 10.9734/AJAHR/2020/v6i230069.

Chapagain TR, Tiwari DN, Adhikari RC, Shrestha MB. 2014. Physicochemical properties and yield of tomato varieties under plastic house condition. Nepal J. Sci. Tech., 15(2): 1722. doi.org/10.3126/njst.v15i2.12106.

Devkota S, Shrestha SL, Dhakal DD, Shakya SM, Pandey J. 2018. Evaluation of tomato hybrids for yield attributes under Khumaltar condition. J. Inst. Agric. Ani. Sci., 35: 191-196.

FAO, 2019. Food and Agriculture Organization of the United Nations. FAOSTAT. http://www.fao.org/faostat/en/No. of data/QC. [Retrieved on December 25, 2020].

Gotame TP, Gautam IP, Shrestha SL, Pradhan NG. 2019. A field guide for vegetable germplasm testing and variety registration/release. Nepal Agricultural Research Council (NARC), National Agriculture Research Institute (NARI), Horticulture Research Division (HRD) Khumaltar, Lalitpur. NARC publication serial No. 00669-683 (2019/20).

Gotame TP, Shrestha SL, Poudel S, Shrestha J. 2021. Growth and yield performance of different open-pollinated tomato genotypes in the Terai region of Nepal. J. Agric. Nat. Res., 4(1): 256-264. doi: https://doi.org/10.3126/janr.v4i1.33280.

Gurung S, Adhikari P, Giri K, Gotame TP, Shrestha SL. 2020. Growth and yield performance of hybrid tomato (Solanum lycopercicum L.) lines at Parwanipur, Bara, Nepal. J. Agric. Natu. Res., 3(1): 180-189. doi: https://doi.org/10.3126/ janr.v3i1.27166.

Hanson P, Lin L, Luther GC, Tsai W, Srinivasan R, Chen C, Lin C, Sheu Z, Lu S. 2013. Procedures for tomato variety field trials. AVRDC Publication No. 11-751. http://203.64. 245.61/fulltext_pdf/FLYER/2011-2015/f0194.pdf and http:// avrdc.org/?page_id=227 [Retrieved on August 20, 2019].

IPGRI, 1996. Descriptors for tomato (Lycopersicon spp.). International Plant Genetic Resource Institute (IPGRI), Rome (Italy). ISBN 10: 92-9043-294-2. https://www. bioversityinternational.org/fileadmin/_migrated/uploads/tx_ news/Descriptors_for_tomato__Lycopersicon_spp._286.pd f. [Retrieved on January 2020].

MoALD, 2019. Statistical information on Nepalese agriculture. Ministry of Agriculture and Livestock Development, AgriBusiness Promotion and Statistics Division, Singh Durbar, Kathmandu, Nepal.

Panthee D, Gotame TP. 2020. Improving heat stress tolerance in tomato. CAB Reviews, 15(060): 1-14. doi: 10.1079/PAVSN NR202015060.

Paudel KB, Gautam IP, Regmi HN, Chaudhary JN, Upadhaya KP, Dauwadi P, Pokharel D, Khatri B. 2004. Evaluation of tomato (Lycopersicum esculentum L.) genotypes in the farmer's field for the rainy season in the western hills of Nepal. Proceeding of the Fourth National Workshop on Horticulture, March 2-4, 2004. Nepal Agricultural Research Council, National Agriculture Research Institute and Horticulture Research Division, Khumaltar, Lalitpur, Pp: 422-426. 
Rawal R, Gautam DM, Gautam IP, Khadka RB, Tripathi KM, Hanson P, Acedo AL, Easdown W, Hughes JA, Keatinge JDH. 2017. Evaluation of AVRDC advanced lines of long shelf life tomato in the Terai region of Nepal. Acta Hortic., 1179: 305-310. doi: org/10.17660/ActaHortic.2017.1179. 47.

Regmi HN, Budathoki K, Pradhan NG. 2004. Evaluation of tomato inbred lines for the production of F1 hybrids. Proceeding of the Fourth National Workshop on Horticulture, March 2-4, 2004. Nepal Agricultural Research Council, National Agriculture Research Institute and Horticulture Research Division, Khumaltar, Lalitpur. pp: 401-404.
Reddy BR, Reddy MP, Reddy DS, Begum H. 2013. Correlation and path analysis studies for yield and quality traits in tomato (Solanum lycopersicum L.). Journal of Agriculture and Veterinary Science, 4(4): 56-59. doi.org/10.9790/23800445659.

Sato S, Peet MM, Thomas JF. 2000. Physiological factors limit fruit set of tomato (Lycopersicon esculentum Mill.) under chronic, mild heat stress. Plant, Cell and Environ., 23(7): 719726.

Shrestha SL, Sah RL. 2014. Evaluation of tomato cultivars for central Terai of Nepal. Nepal J. Sci. and Tech., 15(2): 11-16. 